\title{
Vulnerability of Complex Networks under Approximate Longest Chain Attack Strategies
}

\author{
Yao-Hui HAO ${ }^{1,2, a}$, Jia-Liang SONG ${ }^{1}$, Yi LIN ${ }^{1}$, Ji-Hong HAN ${ }^{1}$ \\ ${ }^{1}$ ZhengZhou Institute of Information Science and technology, Zhengzhou 450001, China \\ 2State Key Laboratory of Mathematical Engineering and Advanced Computing, Zhengzhou 450001, China
}

\begin{abstract}
We proposed three approximate longest chain-attack strategies and studied the vulnerability of complex networks under these intentional attack strategies. Experimental results indicate that when random network and small-world network are under any one of the three chain-attacks, the larger the $\langle k>$ is, the smaller the iterative step $T$ is. However, scale-free network shows different characteristics: the larger the $\langle k>$ is, the larger the $T$ is. These findings supplement and extend the previous attack results on nodes and edges, and can thus help us better explain the vulnerability of different complex networks.
\end{abstract}

\section{Introduction}

Network vulnerability is one of the most important issues of complex networked systems. In recent decades, many researchers proposed many attack strategies and analyzed vulnerability of various complex network models and real world networks[1-4], ranging from biology to Internet $[5,6]$, power to transportation[7-9].

However, when analyzing the vulnerability of the network structure, researchers generally adopted the attack strategy of randomly or intentionally removing a certain percentage of the network nodes or edges, according to selected centrality measurement indicators [13-15] (such as: initial or re-calculated degree and betweenness[10-12]). In addition, it is unnecessary for these removed nodes and edges to be adjacent. But, by analyzing kill chain attack mode $[16,17]$, we find that nowadays attacks often follow two steps: to intrude nodes and to destroy nodes. In the first step, the attackers will invade a network node by implanting the virus program into it, but they won't immediately destroy the node after that. Instead, the attackers continue to invade one adjacent uninvaded node until the largest number of invaded nodes is met. Then, the attackers begin to enter the second phase, namely to destroy the nodes. Once it gets started, all nodes that are implanted with virus would be destroyed at the same time. That is to say, when attackers destroy nodes in the network, the destroyed nodes also present a chain structure way, instead of a discrete distribution way. This is distinctly different from the previous attack strategies.
As such, we proposed three types of approximate longest chain-attacks according to different node selection strategies, and investigated vulnerability of complex networks including three model networks: random network, scale-free network, small-world network and two real world networks. In this paper, the efficiency of chain-attacks is determined by the largest connected subgraph and the number of iterative steps. Note that, all the experiments and algorithms in this paper are based on the assumption that the networks are undirected and there is only one edge between two nodes.

\section{Model of Chain Attack Strategies}

\subsection{Chain Structure of Invasion}

The more nodes are invaded, the more nodes could be destroyed at a time. Thus, each invasion should better achieve the maximum length of the chain. According to the research results of network centrality, the nodes with max degree or max betweenness play an important role in network connectivity[18-20]. So, we propose three approximate longest chain-attack strategies based on these two centrality measures, which are suitable for large-scale attack implementation. The descriptions are as follows:

(1)RLC(random longest chain augmenting approach): First, RLC randomly selects a node in the network as the initial node. Second, RLC randomly chooses a neighboring node of the initial node as the second node of the chain, and then chooses a neighboring node of the second node as the

\footnotetext{
${ }^{a}$ Corresponding author: hao_yaohui@126.com
} 
third node of the chain, and so on [21]. No node should be invaded twice in the chain augmenting process and it continues until no appropriate node could be found.

(2)BLC(longest chain augmenting approach of max betweeness): First, BLC selects the max betweeness node in the network as the initial node, and then chooses the max betweeness node in initial node's neighboring nodes as the second node of the chain, and so on. No node should be invaded twice in the chain augmenting process and it continues until no appropriate node could be found. (If there is more than one max betweeness node, we randomly select a node from them.)

(3)DLC(longest chain augmenting approach of max degree): DLC adopts a similar method as (2), but the difference is DLC selects the nodes with the max degree.

In this paper, Degree of a node $i$ is defined by

$$
k_{i}=\sum_{j=1}^{N} a_{i j}=\sum_{j=1}^{N} a_{j i},
$$

where aij is the element of the adjacency matrix $\mathrm{A}$ in the given network $\mathrm{G}, \mathrm{A}=($ aij $) \mathrm{N} \times \mathrm{N}$.

Analogously, Betweenness of a node $\mathrm{i}$ is defined as the sum of proportions of the number of shortest paths between all pairs of destination nodes that go though node $\mathrm{i}$ :

$$
B_{i}=\sum_{s \neq i \neq t} \frac{n_{s t}^{i}}{g_{s t}},
$$

where gst is the total number of the shortest paths from node s to node $\mathrm{t}$, and $n_{s t}^{i}$ is the number of those that pass through the node i. In a network, Betweenness counts the number of geodesic paths that pass through a node.

An example is given to explain the intrusion process of these three chains, as shown in figure 1.

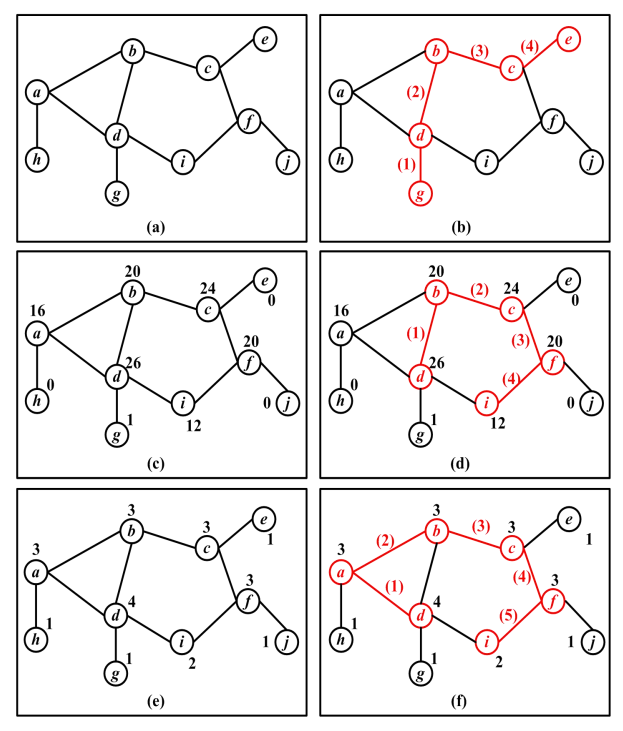

Fig.1. An Example for Intrusion Chain Structure (a) Network topology; (b) Choose chain $\{$ g,d,b,c,e $\}$ by following RLC strategy; (c) Betweenness of each node in the network; (d) Choose chain $\{\mathrm{d}, \mathrm{b}, \mathrm{c}, \mathrm{e}, \mathrm{f}, \mathrm{i}\}$ by following BLC strategy; (e) Degree of each node in the network; (f) Choose chain $\{\mathrm{d}, \mathrm{a}, \mathrm{b}, \mathrm{c}, \mathrm{e}, \mathrm{f}, \mathrm{i}\}$ by following DLC strategy (After the max degree node $\mathrm{d}$ is selected, the degrees of a and $b$ are equal and we randomly select $a$ or $b$ node as the second node of the chain.)

\subsection{Implement and Measure of Chain Attack}

After successful invasion of the nodes, the attackers will implement nodes destruction. Provided that the invaded nodes are completely controlled by the attackers, it is clear that the attackers can easily destroy all the invaded chain nodes. In this paper, we show a node is destroyed by removing it. Obviously, each time the nodes removed is a chain. Furthermore, all edges incident with these removed nodes are also removed.

We use the number of nodes in the largest connected subgraph(S) to measure the vulnerability of different networks [11]. Here, $\mathrm{N}$ is the number of nodes in initial network, $\mathrm{N}^{\prime}$ is the number of nodes in the largest connected subgraph after the attack, i.e. $\mathrm{S}=\mathrm{N}$ '. To compare the initial networks with different number of nodes, we can execute a data reduction on the network size. The formula is as follows.

$$
S^{\prime}=\frac{S}{N}=\frac{N^{\prime}}{N}
$$

The size of $\mathrm{S}$ in a network reflects the communication capability of a network. This means that when the network is attacked, the larger the largest connected subgraph, the more robust the network. If $S^{\prime} \approx 1$, it means that the network is highly connected.

In this paper, the chain-attacks are implemented iteratively, i.e., each time the nodes of a chain are removed until all the nodes in the network are disconnected. The number of iterative steps $(\mathrm{T})$ reflects the tolerance of a network [21]. The initial value of $\mathrm{T}$ is zero, and it increases by one each time with a chain-attack. The greater $\mathrm{T}$ is, the more iteration there is, and the more robust the network is. The specific implementation processes of the chain-attacks are shown in Fig. 2. 


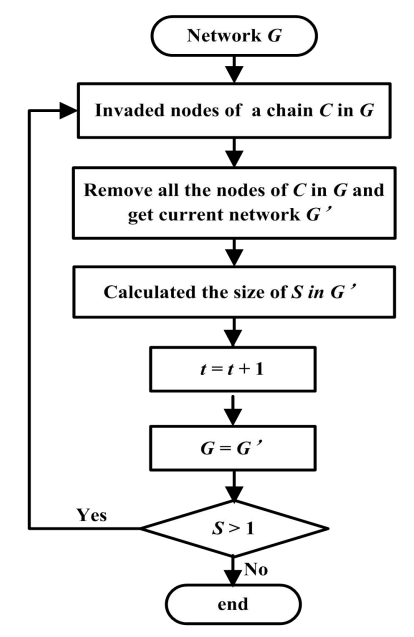

Fig. 2. The Flow Diagram of Chain Attacks

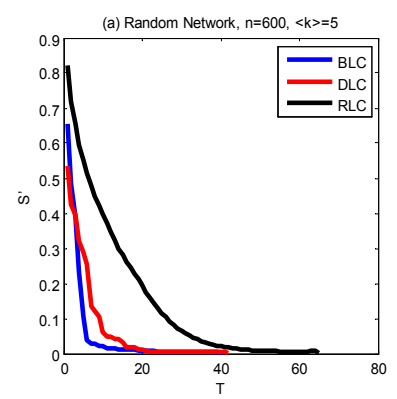

Each iteration indicates implementing a chain-attack, which includes two operations, invading nodes and removing nodes. When the size of the largest connected subgraph in the current network $G$ is 1 , which means all the nodes in $\mathrm{G}$ are isolated, the iteration will then be terminated.

\section{Simulation and Analysis}

In this part, we show the experimental results of three model networks under chain-attacks, including random network,scale-free network, small-world network.

Firstly, we study the change of the largest connected subgraph $\mathrm{S}$ for random, scale-free and small-world model networks, under three different chain-attacks. The change curves of S are shown in Figure 3.
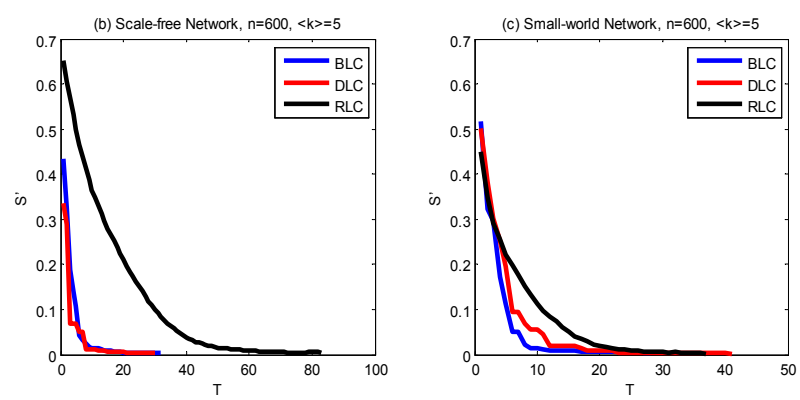

Fig. 3. The Variation of $s$ ' Under Three Chain-Attack Trategies.

(a) S' vs. T in Random Network; (b) S' vs. T in Scale-Free Network; (c) S' vs. T in Small-World Network

As shown in Fig.3(a)(b), in the whole iterative implementation of the chain-attacks for random and scalefree model networks, for a given $t, S(t) R L C$ is larger than $\mathrm{S}(\mathrm{t}) \mathrm{BLC}$ and $\mathrm{S}(\mathrm{t}) \mathrm{DLC}$. This indicates that the curve of $\mathrm{S}(\mathrm{t})$ RLC decreases significantly slower than S(t)BLC and $\mathrm{S}(\mathrm{t}) \mathrm{DLC}$, namely, DLC and BLC chain-attack strategies are more destructive than RLC chain-attack strategy for three model networks.

Moreover, for random network, we can see two crosses $(\mathrm{t}=3, \mathrm{t}=20)$ between DLC and BLC chain-attack strategies in Fig.3(a). When $t \leq 3$, BLC has similar destructive effect to DLC; while when $3 \leq \mathrm{t} \leq 20$, BLC can perform more destructive effect than DLC; as $\mathrm{t} \geq 20$, BLC has the similar destructive effect to DLC again. And the max iterative steps are ranked as follows:

$\operatorname{MAX}(\mathrm{T}) \mathrm{RLC}>\operatorname{MAX}(\mathrm{T}) \mathrm{DLC}>\operatorname{MAX}(\mathrm{T}) \mathrm{BLC}$.
As for scale-free network in Fig.3(b), the change curves of $\mathrm{S}$ are almost the same for BLC and DLC. Though, DLC has a more destructive effect at the beginning.

For small-world network (Fig.3(c)), there are two crosses $(t=3, t=25)$ in three curves. While $t \leq 3$, the three chain-attack strategies have the similar destructive effect on the small-world network. However, when $3 \leq t \leq 25$, BLC has the greatest destructive effect in these chain-attack strategies. In the latter fraction of the attacks, while $t \geq 25$, the changes of three curves are similar again, and the max iterative steps are ranked as follows: $\operatorname{MAX}(\mathrm{T}) \mathrm{DLC}>\operatorname{MAX}(\mathrm{T}) \mathrm{BLC}>\operatorname{MAX}(\mathrm{T}) \mathrm{RLC}$. So, the efficiency of the chain-attack strategies is BLC $>$ DLC $>$ RLC for these three mode networks.

Secondly, we compare changes of the largest connected subgraphs S' of random, scale-free and small-world model networks under three chain-attacks, as shown in fig. 4 . 

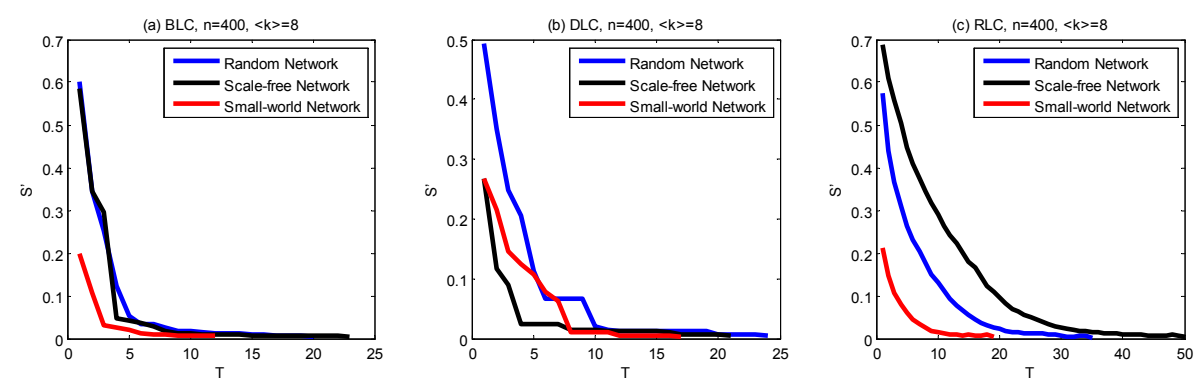

Fig. 4. $S$ ' vs. $T$ (a) BLC; (b) DLC; (c) RLC

From Fig.4(a), we can see that the curves of random and scale-free networks are very similar under the BLC attack strategy, and for a given $t$, the $S(t)$ of small-world is the lowest. The max iterative step of three model networks has the following relationship: $\operatorname{MAX}(\mathrm{T}) \mathrm{SF}>\operatorname{MAX}(\mathrm{T}) \mathrm{RN}>$ $\operatorname{MAX}(\mathrm{T}) \mathrm{SW}$, namely small-world network is most vulnerable under BLC chain-attack.

In Fig.4(b), under the DLC attack, the curve of Scalefree network has the fastest decline rate in the first fraction of three curves $(\mathrm{t} \leq 8)$; while $\mathrm{t}>8$, for a given $\mathrm{t}$, $\mathrm{S}$ has the following relationship: $\mathrm{S}(\mathrm{t}) \mathrm{RN}>\mathrm{S}(\mathrm{t}) \mathrm{SF}>\mathrm{S}(\mathrm{t}) \mathrm{SW}$, and the max iterative step has the following relationship: $\operatorname{MAX}(\mathrm{T}) \mathrm{RN}>\operatorname{MAX}(\mathrm{T}) \mathrm{SF}>\operatorname{MAX}(\mathrm{T}) \mathrm{SW}$. It means that scale-free network is the most vulnerable and random network is the most robust in the first fraction of DLC chain-attack. But, in the latter part, small-world network becomes the most vulnerable and random network is still the most robust. The vulnerability of scale-free network is between random and small-world network.

Fig.4 (c) shows that under the RLC attack, when the value of $\mathrm{T}$ is a given $\mathrm{t}, \mathrm{S}(\mathrm{t}) \mathrm{SF}>\mathrm{S}(\mathrm{t}) \mathrm{RN}>\mathrm{S}(\mathrm{t}) \mathrm{SW}$, and the max iterative step has the following relationship: $\operatorname{MAX}(\mathrm{T}) \mathrm{SF}>\operatorname{MAX}(\mathrm{T}) \mathrm{RN}>\operatorname{MAX}(\mathrm{T}) \mathrm{SW}$. It shows that scale-free network has the best robustness, while smallworld network has the weakest robustness, and the robustness of random network is in the middle.

And in Fig.4(a)(b)(c), for Random, scale-free and smallworld model networks under three chain-attacks, all curves decrease rapidly in the first fraction and slowly in the latter part.

Lastly, we analyze change of the largest connected subgraph $\mathrm{S}$ of three modes networks, under these three chain-attacks with different network density. The analysis results are shown in fig.5.

Under the BLC, DLC and RLC chain-attacks, random network (Fig.5(a)(b)(c)) shows that the larger $<\mathrm{k}>$ is, the smaller MAX (T) is. In general, the larger $<k>$ is, the faster $S$ ' declines. It indicates that the robustness of random network grows in inverse proportion to $<\mathrm{k}>$ under these three chain-attacks. scale-free network (Fig.5(d)(e)(f)) shows that the larger $<k>$ is, the larger $\operatorname{MAX}(\mathrm{T})$ is. It means that the robustness of scale-free network is in proportion to $<\mathrm{k}>$ under chain-attacks. small-world network (Fig.5(g)(h)(i)) has shown a similar performance to the random network, that is, there is an inverse proportion between the robustness of small-world network and the size of $<\mathrm{k}>$ under chain-attacks.

In addition, we calculate the average distance of random, scale-free and small-world networks under different densities $<\mathrm{k}>$. As can be seen from Table 1, for random network and small-world network, the greater the $<\mathrm{k}>$ is, the smaller the average distance is, but for the scale-free network, the opposite is the case. We also analyze the number of removed nodes at each iterative step of the chain-attacks, as shown in Fig. 6. 

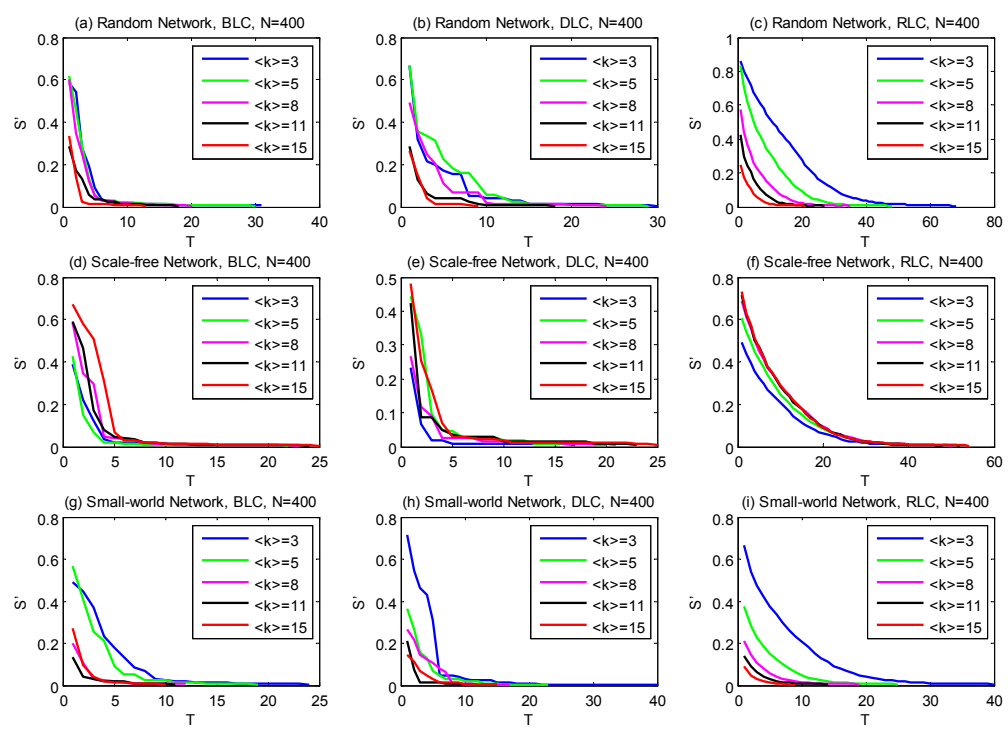

Fig.5. $S$ 'vs. $T, N=400$
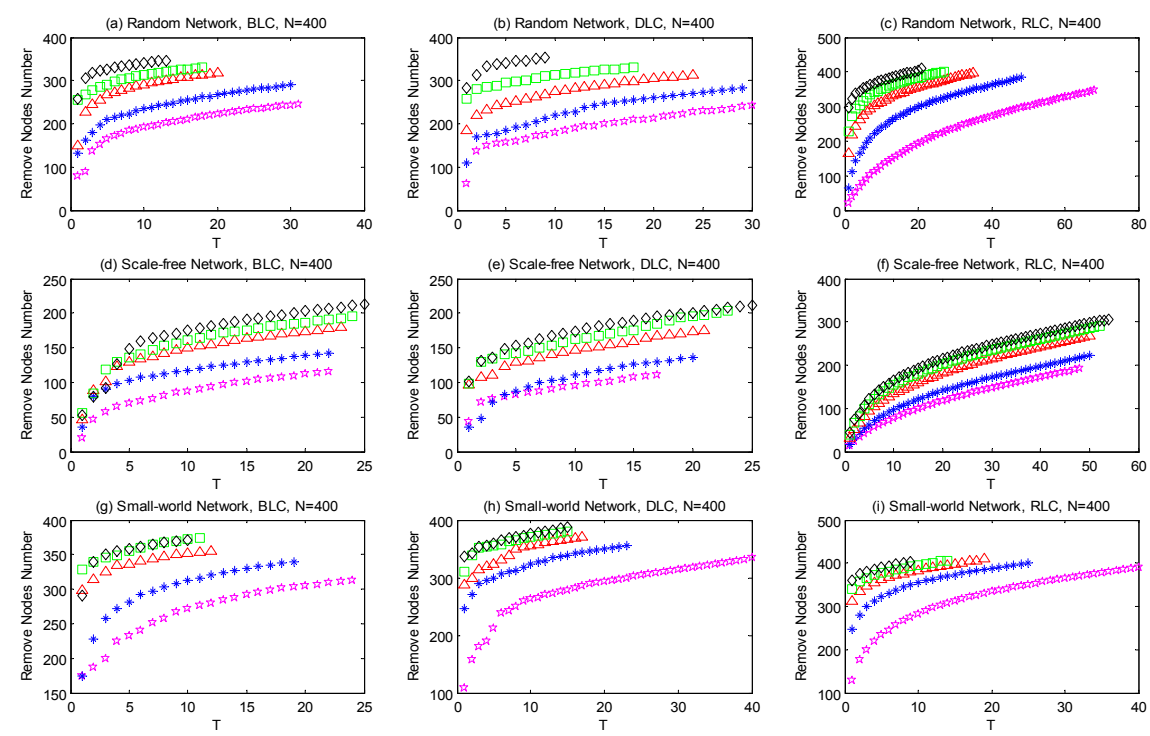

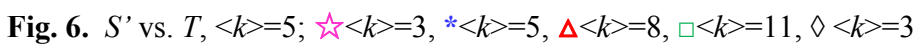

As can be seen from Fig. 6, for three model networks at the same iterative step $t$, the number of nodes removed increases accordingly as $<\mathrm{k}>$ increases. Combining the average distance of model networks under different $<\mathrm{k}>$ (Table 1), we can ascertain that the chain-attack is more efficient as the increasing $<\mathrm{k}>$ reduces the average distance among nodes in the random network and small-world network. So for the random network and small-world network under the chain-attacks, the larger the $<\mathrm{k}>$ is, the smaller the $\operatorname{MAX}(\mathrm{T})$ is.

Table 1. The Average Distance of Networks

\begin{tabular}{|c|c|c|c|}
\hline & random & scale-free & small-world \\
\hline$<k>=3$ & 4.6539 & 1.2662 & 3.6371 \\
\hline
\end{tabular}




\begin{tabular}{|l|l|l|l|}
\hline$<k>=5$ & 3.9037 & 1.5523 & 2.9277 \\
\hline$<k>=8$ & 3.1518 & 2.1086 & 2.5215 \\
\hline$<k>=11$ & 2.7787 & 2.3257 & 2.2879 \\
\hline$<\mathrm{k}>=15$ & 2.5149 & 2.4682 & 2.0719 \\
\hline
\end{tabular}

\section{Conclusions}

In this study, we research on the vulnerability of complex network against new-type intentional attack strategies close to the real attack behavior. In sum, we analyze the vulnerability of some complex networks by iteratively implementing three different chain-attacks, RLC, BLC and DLC. We obtain that when random network and smallworld network are under any one of the three chain-attacks, the larger the $\langle k\rangle$ is, the smaller the $\mathrm{T}$ is. However, scalefree network shows different characteristics: the larger the $<k>$ is, the larger the $\mathrm{T}$ is. These findings supplement and extend the previous attack results and can thus help us to provide some insights into the design of more robust networks.

\section{References}

[1] M. Bellingeri, D. Cassi, S. Vincenzi, Phys. A 414, 174 (2014).

[2] A. Yehezkel, R. Cohen, PHYS. R. E 86 (2012).

[3] H. P. Ren, J. Song, R. Yang, M. S. Baptista, C. Grebogi, Phys. A 442, 239 (2016).

[4] B. P. Duan, J. Liu, M.X. Zhou, L. L. Ma, Phys. A 448, 144 (2016).

[5] R. Albert, H. Jeong, A. L. Barabasi, Nature 401, 307 (1999).

[6] S. N. Dorogovtsev, J. F. Mendes, Evolution of Networks: From Biological Nets to the Internet and $W W W$ (Oxford University Press, 2013).

[7] R. Albert, I. Albert, G. L. Nakarado, Phys. Rev. E 69 (2004).

[8] M. Ouyang, L. J. Zhao, Z. Z. Pan, L. Hong, Phys. A 403, 45 (2014).

[9] H. Demirel, M. Kompil, F. Nemry, Trans. R. P. A 81, 62 (2015).

[10] R. Albert, H. Jeong, A. L. Barabasi, Nature 406, 387 (2000).

[11] P. Holme, B. J. Kim, C. N. Yoon, S. K. Han, Phys. Rev. E 65 (2002).

[12] T. Y. Nie, Z. Guo, K. Zhao, Z.M. Lu, Phys. A 424, 248 (2015).

[13] J. H. Zhang, L. Hong, S. L. Wang, IEEE International Conference on Service Operations, (2011).

[14] M. E. J. Newman, S. H. Strogatz, D. J. Watts, Phys. Rev. E. 64 (2001).

[15] W. J. Xiao, L. X. Lin, G. R. Chen, Phys. A 437, 437 (2015).

[16] A. K. Sood, R. J. Enbody, Proceedings of IEEE Security and Privacy Magazine 11, 54 (2013).
[17] F. Baiardi, F. Tonelli, A. D. R. D. Biase, 24th Euromicro International Conference on Parallel, Distributed, and Network-Based Processing (2016).

[18] J. W. Wang, L. L. Rong, Safety Science 47, 1331 (2009).

[19] J. Ugander, B. Karrer, L. Backstrom, C. Marlow, arXiv (2011).

[20] J. Hendler, N. Shadbolt, W. Hall, T. B. Lee, D. Weitzner, Communications of the ACM 51, 60 (2008).

[21] C. L. Pu, W. Cui, Phys. A 419, 622 (2015). 